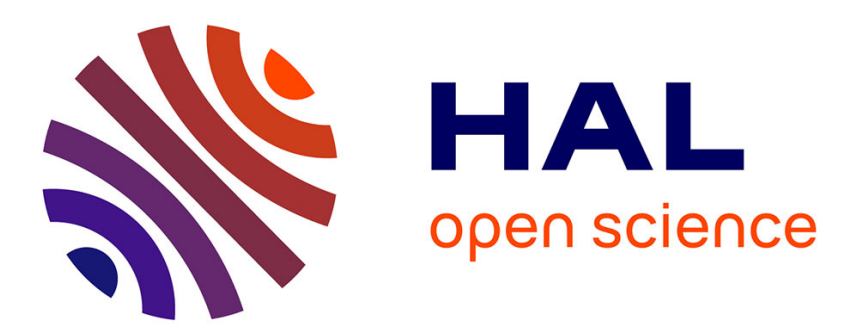

\title{
High-resolution, large-dynamic-range multimode interferometer sensor based on a suspended-core microstructured optical fiber
}

Yu Zheng, Perry Ping Shum, Yiyang Luo, Yanan Zhang, Wenjun Ni, Guanghui Wang, Zhifang Wu, Xuan Quyen Dinh, Jean-Louis Auguste, Georges Humbert

\section{To cite this version:}

Yu Zheng, Perry Ping Shum, Yiyang Luo, Yanan Zhang, Wenjun Ni, et al.. High-resolution, largedynamic-range multimode interferometer sensor based on a suspended-core microstructured optical fiber. Optics Letters, 2020, 45 (4), pp.1017. 10.1364/OL.386296 . hal-02525003

\section{HAL Id: hal-02525003 \\ https://hal.science/hal-02525003}

Submitted on 14 Dec 2020

HAL is a multi-disciplinary open access archive for the deposit and dissemination of scientific research documents, whether they are published or not. The documents may come from teaching and research institutions in France or abroad, or from public or private research centers.
L'archive ouverte pluridisciplinaire HAL, est destinée au dépôt et à la diffusion de documents scientifiques de niveau recherche, publiés ou non, émanant des établissements d'enseignement et de recherche français ou étrangers, des laboratoires publics ou privés. 


\title{
High-resolution, large dynamic range multimode interferometer sensor based on a suspended core microstructured optical fiber
}

\author{
Yu Zheng, ${ }^{1,2}$ Perry Ping Shum, ${ }^{1,2, *}$ Yiyang Luo, $1,2,8$ Yanan Zhang, ${ }^{3}$ Wenjun \\ NI, ${ }^{1,2}$ GuanghuI Wang, ${ }^{4}$ Zhifang Wu, ${ }^{5}$ XUAN QuYen Dinh, ${ }^{2,6}$ Jean-LouIS \\ Auguste, ${ }^{7}$ Georges Humbert, ${ }^{7}$
}

\author{
${ }^{1}$ School of Electrical and Electronic Engineering, Nanyang Technological University, 639798, Singapore \\ ${ }^{2}$ CINTRA CNRS/NTU/THALES, UMI 3288, Research Techno Plaza, 50 Nanyang Drive, 637553, Singapore \\ ${ }^{3}$ College of Information Science and Engineering, Northeastern University, Shenyang 110819, China \\ ${ }^{4}$ College of Engineering and Applied Sciences, Nanjing University, Jiangsu 210093, China \\ ${ }^{5}$ College of Information Science and Engineering, Huaqiao University, Xiamen, 361021, China \\ ${ }^{6} R \& T$, Thales Solutions Asia Pte Ltd., Singapore 498788, Singapore \\ ${ }^{7} X$ LIM Research Institute, UMR 7252 CNRS/University of Limoges, Limoges, 87060, France \\ ${ }^{8}$ e-mail: luoyy@ntu.edu.sg \\ *Corresponding author: yrrepaper@outlook.com
}

Received XX Month XXXX; revised XX Month, XXXX; accepted XX Month XXXX; posted XX Month XXXX (Doc. ID XXXXX); published XX Month XXXX

The performance of sensors, including optical fiber sensors, are commonly limited by the trade-off between a large dynamic range and a high resolution. Here, in order to optimize both, we propose an inline multimode interferometer sensor based on a suspended core microstructured optical fiber. Due to the existence of multiple pairs of mode interferences, the transmission spectrum of the interferometer consists of dense fringes modulated by a lower envelope. Since these mode interferences take place in the uniform material with the same length, the dense fringes and the lower envelope have an identical sensing response without crosstalk. Hence, the sensor integrates the large dynamic range of the lower envelope and the high resolution of the dense fringes. Strain sensing performance is investigated to validate the characteristic of the large dynamic range and high resolution of the proposed sensor. The dynamic range, theoretically $0-9200 \mu \varepsilon$, is 12 times larger than the dense fringes and the resolution is $\mathbf{1 7 . 5}$ times higher than the lower envelope. America

(C) 2019 Optical Society of

http://dx.doi.org/10.1364/OL.99.099999

Optical fiber sensors have been widely investigated due to their advantages of easy integration, high stability, repeatability and electromagnetic immunity[1-4]. However, the performance of these sensors is limited by either their dynamic range or resolution, which is a trade-off existing in almost all kinds of sensor. Liu et al. developed an optical fiber thermometer with double-cascaded silicon cavities, one thin and one thick, to tackle this problem [5]. The thin cavity provides the ability of large dynamic range measurement, and the thick cavity defines the high resolution. Nevertheless, the fabrication of ultra-thin cavities (10 and $200 \mu \mathrm{m})$ is complex.

Strain sensors, as one of the most important optical fiber sensors, have widespread applications in many fields, such as geophysics research, mechanical engineering and particularly in the field of structural health monitoring for bridges, towers, railways et al [68]. Various types of schemes have been used to demonstrate the optical fiber strain sensors, for instance, a polarization-maintaining photonic crystal fiber (PCF) inserted in a Sagnac loop [9], a fiber Bragg grating (FBG) in a microstructured optical fiber (MOF) [10], a Pound-Drever-Hall feedback loops based on a FBG and a FabryPerot interferometer (FPI) [11] and an in-line Mach-Zehnder interferometer in twisted multicore fiber [12]. However, as we mentioned above, the performance of these sensors is restricted by the trade-off between the dynamic range and resolution. For example, the resolution of the sensor based on Sagnac interferometer is limited at $43 \mu \varepsilon$ with a dynamic range of $32 \mathrm{m \varepsilon}$; the sensor fabricated by inscribing the FBG into the MOF only possesses the dynamic range of around $430 \mu \varepsilon$ with a resolution of $8.6 \mu \varepsilon$; the sensor composed of the FBG and the FPI has ultrahigh resolution $(0.01 \mathrm{n} \varepsilon)$, but the dynamic range of $300 \mu \varepsilon$ is narrow. Meanwhile, the secondary processing inside the specialty fibers, such as inscribing gratings or twisting fibers, will improve the 
complexity of the fabrication procedure and reduce the repeatability of the fiber sensors.

Suspended core microstructured optical fiber (SCMOF), as a typical MOF, is composed of a triangular core suspended with three thin struts and three air holes surrounding the fiber core. Due to the large refractive index (RI) difference between fiber core and cladding in the SCMOF, high order modes are easily excited in the small area of suspended triangular core [13]. Meanwhile, a high numerical aperture (NA) caused by the large RI difference leads to a low bending loss for high order modes. Hence, SCMOF can serve as an ideal platform for developing fiber sensors based on multimodal interference.

In this paper, we theoretically and experimentally demonstrated an inline multimode interferometer sensor based on a SCMOF with a large dynamic range and a high resolution. The multimode interferometer sensor was fabricated only by splicing a section of SCMOF between two segments of single mode fibers with central alignment. The transmission spectra of SMF-SCMOF-SMF structure features dense fringes modulated by a lower envelope, which is induced by the multimode interferences. The theoretical model and experimental results match well. The strain sensing performance of sensors with different lengths SCMOF was investigated. Our scheme can be applied to fabricate the strain sensors with a particular resolution, dynamic range or sensitivity by adjusting the length of SCMOF.

The cross-sectional image of the SCMOF illustrated in Fig. 1(a) was obtained with a scanning electron microscope (SEM). The fiber was fabricated with pure silica tubes by appling the stack-and-draw process. It is composed of a triangular fiber core surrounded by three crown-like air holes. The diameter of the circle inscribed in the triangular core is $3.3 \mu \mathrm{m}$. The diameter of air cladding is $77 \mu \mathrm{m}$, and the diameter of the fiber is $118 \mu \mathrm{m}$. The thickness of three struts is about $750 \mathrm{~nm}$.

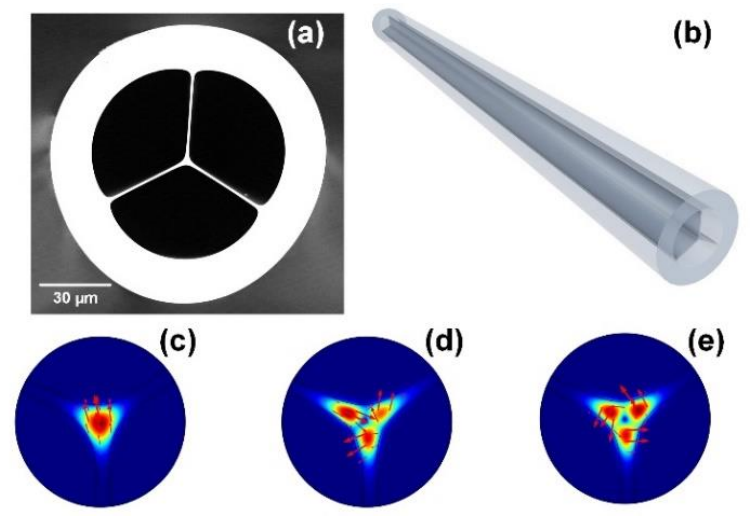

Fig. 1. (a) SEM image of the SCMOF cross section; (b) schematic diagram of the SCMOF; simulated mode field distributions of (c) LP $\mathrm{P}_{01}$ mode, (d) first high order mode, (e) second high order mode at the wavelength of $1550 \mathrm{~nm}$

For theoretically studying the transmission spectra of SCMOF, the transverse mode profiles of SCMOF were simulated by COMSOL Multiphysics 5.0. In the simulation model, the geometry of SCMOF was imported from its SEM image. The results indicated that the SCMOF steadily supported $\mathrm{LP}_{01}$ mode and two high order modes with different mode field distributions propagating in the suspended core, as shown in Fig. 1 (c)-(e). When multiple modes were excited and propagated in the triangular fiber core, interference would take place and lead to a transmission spectrum obtained by the fellow equation:

$$
\begin{aligned}
& I(\lambda)=2 \mathrm{I}_{1}(\lambda)+2 \mathrm{I}_{2}(\lambda)+2 \mathrm{I}_{3}(\lambda) \\
& +2 \sqrt{\mathrm{I}_{1}(\lambda) \mathrm{I}_{2}(\lambda)} \cos \left(2 \pi \Delta \mathrm{n}_{12} \mathrm{~L} / \lambda\right) \\
& +2 \sqrt{\mathrm{I}_{1}(\lambda) \mathrm{I}_{3}(\lambda)} \cos \left(2 \pi \Delta \mathrm{n}_{13} \mathrm{~L} / \lambda\right) \\
& +2 \sqrt{\mathrm{I}_{2}(\lambda) \mathrm{I}_{3}(\lambda)} \cos \left(2 \pi \Delta \mathrm{n}_{23} \mathrm{~L} / \lambda\right)
\end{aligned}
$$

where $I_{1}(\lambda), I_{2}(\lambda), I_{3}(\lambda)$ are the light intensities of three modes, respectively. $\Delta n_{12}$ is the difference between effective refractive indexes (ERIs) of fundamental mode and first high order mode, $\Delta n_{13}$ is the difference between ERIs of the fundamental mode and the second high order mode and $\Delta n_{23}$ is the difference between ERIs of the first high order mode and the second high order mode. $L$ is the length of SCMOF.

The light intensities of three modes could be obtained by calculating their Poynting vector according to the equation $S=E \times H$. $E$ and $H$, the electric field vector and magnetic field vector respectively, could be achieved under different wavelengths by the simulation in COMSOL. Based on the light intensities and the ERIs of three modes obtained from simulation, the transmission spectra with different SCMOF lengths of $4.0 \mathrm{~cm}, 6.5 \mathrm{~cm}$ and $15.0 \mathrm{~cm}$ were calculated and illustrated in Fig. 2, which was characterized by dense interference fringes modulated by one lower envelope. The free spectrum ranges (FSRs) of the dense interference fringes were $2.67 \mathrm{~nm}, 1.65$ and $0.74 \mathrm{~nm}$ around $1550 \mathrm{~nm}$, respectively, and the FSR of the lower envelope is $32.8 \mathrm{~nm}, 20.4 \mathrm{~nm}$ and $8.9 \mathrm{~nm}$, respectively.

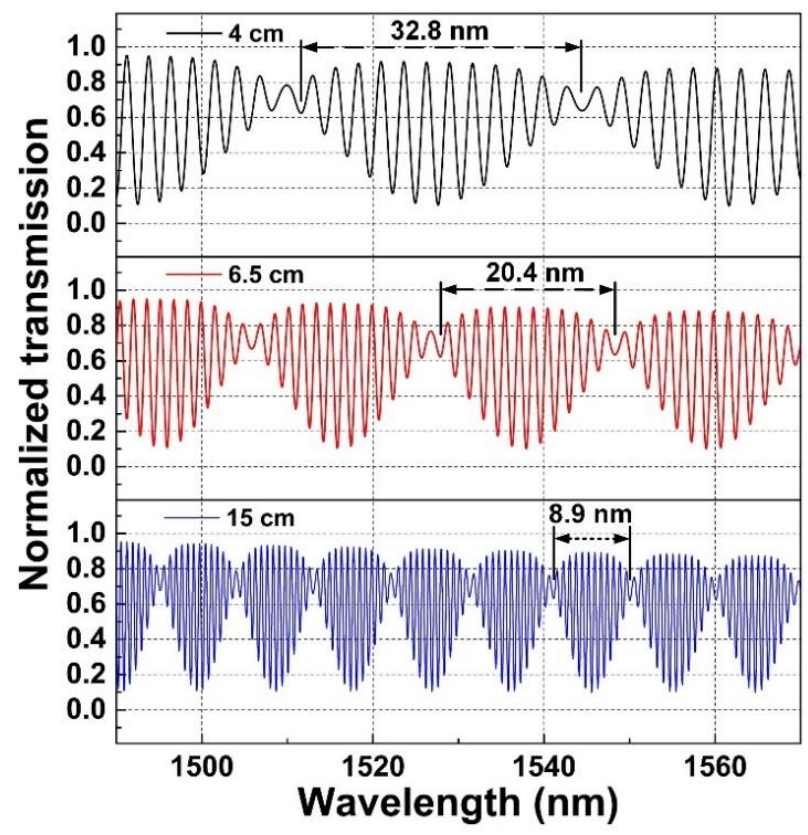

Fig. 2. Simulated interference spectra of SCMOFs with a length of $4.0 \mathrm{~cm}$, $6.5 \mathrm{~cm}$ and $15 \mathrm{~cm}$, respectively.

When axial strain $\varepsilon$ is applied to the SCMOF, the length of SCMOF and the ERI of fiber core will be changed. $\varepsilon$ is defined as $\varepsilon=\Delta L / L 0$, where $\Delta L$ is the change of the fiber length and $L_{0}$ is the initial length of fiber. Since the axial strain is uniform along the fiber, the wavelength shift induced by strain can be calculated by [14]:

$$
\Delta \lambda=-\left(1+2 v+p_{\varepsilon}\right) \varepsilon \lambda
$$


where $p_{\varepsilon}=-n_{0}^{2}\left[(1-v) p_{12}-v p_{11}\right] / 2$ is the effective photoelastic coefficient, $p_{11}$ and $p_{12}$ are the Pockel's coefficients, $v$ is the Poisson ration of the SCMOF, $n_{0}$ is the original RI of fiber core before strain. The equation indicates that the strain response of the sensor is theoretically linear.

The optical characterization of the multimode interferometer was investigated by sandwiching a section of SCMOF into two segments of single mode fibers (SMFs). The alignments between SCMOF and two SMFs were without offset. The splicing machine was Fujikura FSM-100P+, and the arc-time and arc-power are 900 $\mathrm{ms}$ and 210 bits, respectively, which can ensure the repeatability of the device. Three samples have been fabricated with the length of SCMOF $L_{0}=4.0 \mathrm{~cm}, L_{0}=6.5 \mathrm{~cm}, L_{0}=15.0 \mathrm{~cm}$, respectively. The broadband light was launched into the sample from a light source (Infinon Research 1RBL-11111-F), then detected at the sample output by an Optical Spectrum Analyzer (OSA).

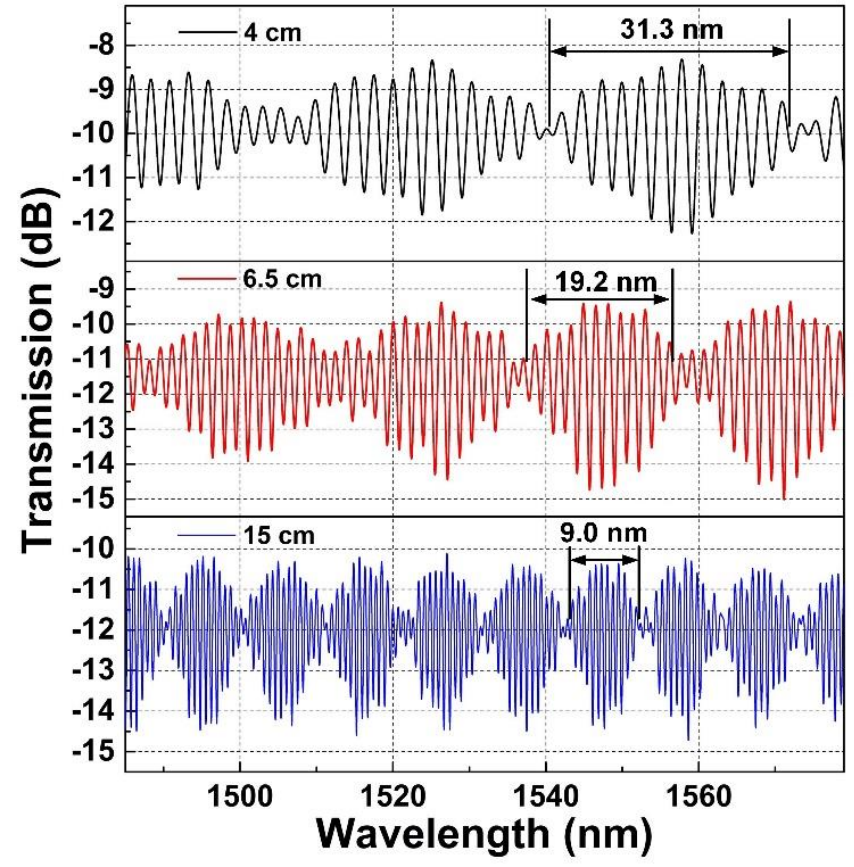

Fig. 3 Experimental transmission spectrum of sensing units with a 4.0 $\mathrm{cm}, 6.5 \mathrm{~cm}$ and $15.0 \mathrm{~cm}$ long SCMOF, respectively.

The FSRs of the dense interference fringes of the sensor with the 4-cm-long, 6.5-cm-long and 15-cm-long SCMOF are $2.57 \mathrm{~nm}, 1.63$ $\mathrm{nm}$ and $0.76 \mathrm{~nm}$ around $1550 \mathrm{~nm}$, respectively. The lower envelopes have a period of $31.3 \mathrm{~nm}, 19.2 \mathrm{~nm}$ and $9.0 \mathrm{~nm}$, respectively. Both experimental FSRs of the dense fringes and the lower envelopes match well with the simulation result, which indicates that the multimode interference proposed in the simulation model dominates the light guiding mechanism of our device.

For further validating the multimode interference in SCMOF, the Fast Fourier Transform (FFT) is adopted to obtain the spatial frequency spectra of the simulation and experiment transmission spectra of the sensor with a 6.5-cm-long SCMOF, respectively. As illustrated in Fig. 4(a), three peaks, $0.05 \mathrm{~nm}^{-1}, 0.59 \mathrm{~nm}^{-1}$ and $0.64 \mathrm{~nm}^{-}$ 1 , are observed in the frequency spectrum of the simulation spectrum, corresponding to the three pairs of mode interferences. The frequency spectrum of the experiment spectrum is plotted in Fig. 4(b). Obviously, the experiment spectrum has same frequency components (Peak 1 and Peak area 2) with the simulation spectrum. Although, several other frequency peaks appear in Fig. 4(b), which should be induced by the mode interferences between higher modes, Peak area 2 dominates in the frequency spectrum. For further investigating the influence of Peak 1 and Peak area 2 on transmission spectrum, the FFT bandpass filtering method is applied to obtain the spatial distribution of dominated mode interferences. Firstly, the other frequency peaks are filtered and Peak 1 and Peak area 2 are kept. Then taking IFFT for the remaining frequency spectrum, the corresponding interference spectrum is displayed in the inset of Fig. 4(b). The interference spectrum reveals neater spectrum distribution than the experiment spectrum in Fig. 3 , and possesses similar distribution with the simulation spectrum in Fig. 2. Hence, the multimode interference in SCMOF induced by three pairs of mode interferences is confirmed.

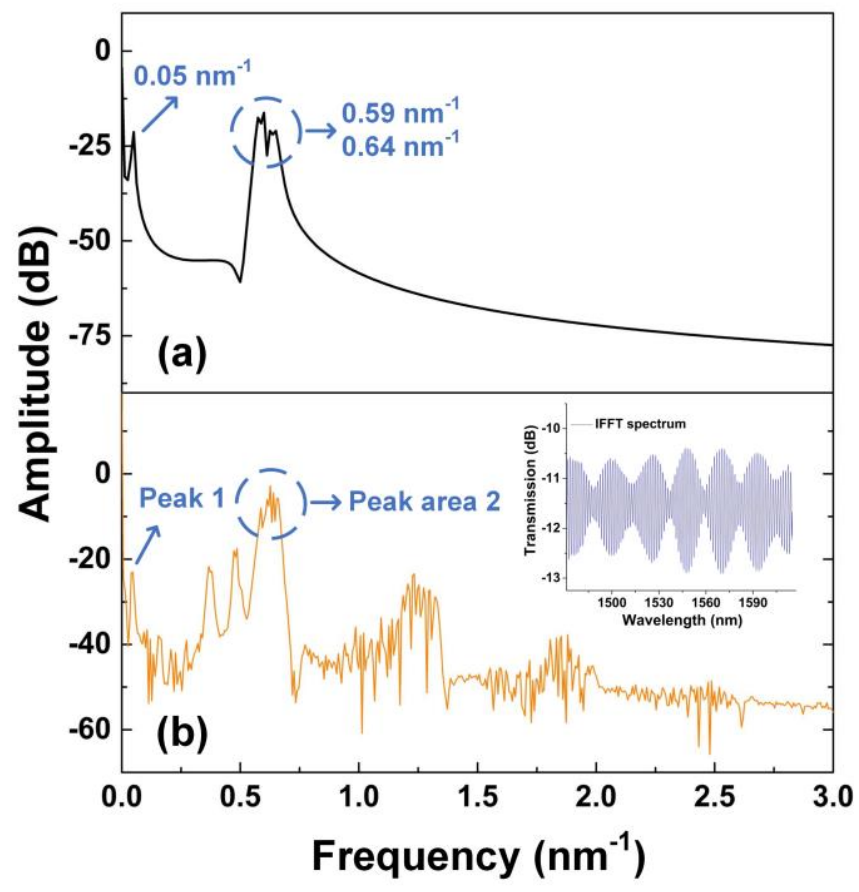

Fig. 4 (a) FFT of the simulation spectrum of the sensor with a $6.5-\mathrm{cm}-$ long SCMOF; (b) FFT of the experiment spectrum of the sensor with a 6.5-cm-long SCMOF, and the inset is the IFFT of the Peak 1 and Peakarea 2.

To validate the large dynamic range and high resolution of the proposed sensor, the devices with a $4.0-\mathrm{cm}$-long length of SCMOF and a $6.5-\mathrm{cm}$-long length of SCMOF were applied to measure the strain response, respectively. The device was straightly hold on two stages with a distance of about $250 \mathrm{~mm}$. Among two stages, one stage was fixed, and the other one was moved to the opposite direction for stretching the device. The strain response performance of our devices are investigated and illustrated in Fig. 5 (a) and (c). The dense interference fringes and lower envelope shift to shorter wavelengths with the axial strain increasing from 0 to $640 \mu \varepsilon$. Compared with the transmission spectrum without strain, the number of their dense fringes in one hump of the lower envelope is equivalent, which indicates that both the dense fringes and the lower envelope have the equal strain responsivity. This is because that three pairs of multimode interferences simultaneously take place in the same fiber core. This spectrum characteristic of our 
device makes the dense fringes and the lower envelope without crosstalk. By using the method of averaging wavelengths of numerous dense peaks, the dynamic range of the sensor will rely on the dynamic range of the lower envelope that is much larger than the dynamic range of the dense fringes [15]. In the meantime, the resolution of the sensor will depend on the resolution of the dense fringes, which is also improved relative to the resolution of the lower envelope. For the sensor we fabricated, the average wavelength positions of the 12 peaks were used to calibrate the strain response, and have been linearly fitted as shown in Fig 5(b) and (d). The linear response is consistent with the theoretical prediction. The corresponding strain sensitivities are calculated to be $-3.4 \mathrm{pm} / \mu \varepsilon$ and $-2.0 \mathrm{pm} / \mu \varepsilon$ for the device with a 4-cm-long SCMOF and a 6.5-cm-long SCMOF, respectively, which indicates that the scheme can be applied to fabricate the strain sensor with a particular sensitivity by adjusting the length of SCMOF. Theoretically, for the sensor with the 4-cm-long SCMOF, the dynamic range of the strain sensor depends on the distance between two peaks of lower envelope and reaches 0-9200 $\mu \varepsilon$, which is 12 times larger than the dynamic range of the dense interference peak $(0-750 \mu \varepsilon)$. Meanwhile, the resolution of the strain sensor with the 4-cm-long SCMOF relies on the full width at half maximum of the dense interference peak and is about 17.5 times higher than that of the lower envelope. The continuous spectral measurement was applied to ensure the method of tracking the set of 12 peaks without ambiguity.
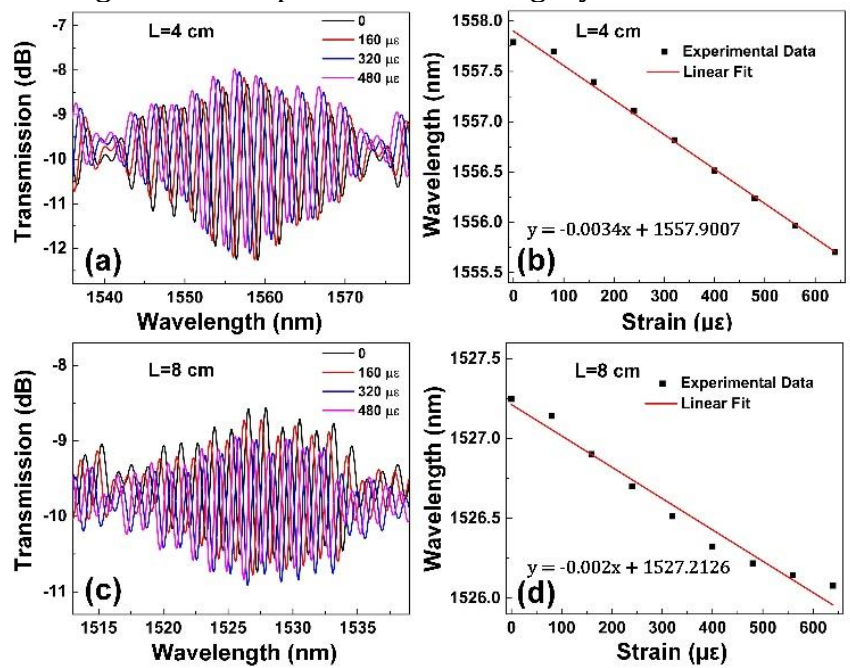

Fig. 5 Spectral evolution of sensing unit with (a) a $4.0 \mathrm{~cm}$ long SCMOF, and (c) a $6.5 \mathrm{~cm}$ long SCMOF for strain measurement; (b) and (d) linear sensitivity variations of from 0 to $640 \mu \varepsilon$.

In summary, an inline multimode interferometer based on the SCMOF has been demonstrated to obtain a large dynamic range and a high resolution simultaneously. The interference spectrum features dense fringes modulated by a lower envelope without ambiguity. The lower envelope determines the large dynamic range, while the dense fringes defines the high resolution. Experimentally, the interferometers with a 4-cm-long, 6.5-cm-long and 15-cm-long SCMOF have been fabricated and characterized. For validating the large dynamic range and high resolution of the interferometer, the strain responses of the sensors with a 4-cm-long and $6.5-\mathrm{cm}$-long SCMOF have been investigated, respectively. Particularly, the dynamic range of the strain sensor with a 4-cmlong SCMOF increases 12 times and the resolution improves 17.5 times. The proposed inline multimode interferometer provides a simple method to tackle the trade-off between a large dynamic range and a high resolution.

Funding. National Research Foundation Singapore (NRF) (NRFCRP18-2017-02), National Natural Science Foundation of China (11774102), the Scientific Research Funds and Promotion Program for Young and Middle-aged Teacher in Science \& Technology Research of Huaqiao University (ZQN-YX504, 17BS412), Open Fund of IPOC (BUPT), Singapore Ministry of Education Academic Research Fund Tier 1 (RG89/16), and MERLION PhD project funded by the Embassy of France in Singapore.

Disclosures. The authors declare no conflicts of interest.

\section{References}

1. K. Li, N. Zhang, N. M. Y. Zhang, G. Liu, T. Zhang, and L. Wei, Opt. Lett. 43, 679-682 (2018)

2. N. Zhang, G. Humbert, T. Gong, P. P. Shum, K. Li, J.-L. Auguste, Z. Wu, D. J. J. Hu, F. Luan, Q. X. Dinh, M. Olivo, and L. Wei, Sensors and Actuators B: Chemical 223, 195-201 (2016).

3. Y. Zheng, Z. Wu, P. Ping Shum, Z. Xu, G. Keiser, G. Humbert, H. Zhang, S. Zeng, and X. Quyen Dinh, Opto-Electronic Advances 1, 1800150118001510 (2018).

4. Y. Zheng, P. P. Shum, S. Liu, B. Li, Y. Xiang, Y. Luo, Y. Zhang, W. Ni, Z. Wu, and X. Q. Dinh, Opt. Express 27, 30629-30638 (2019).

5. G. Liu, Q. Sheng, W. Hou, and M. Han, Opt. Lett. 41, 5134-5137 (2016).

6. Q. Liu, T. Tokunaga, and Z. He, Opt. Lett. 36, 4044-4046 (2011).

7. X. Ye, Y. Su, and J. Han, Sci. World J. 2014, 1-11 (2014).

8. T. H. Chan, L. Yu, H.-Y. Tam, Y.-Q. Ni, S. Liu, W. Chung, and L. Cheng, Engineering structures 28, 648-659 (2006).

9. X. Dong, H. Y. Tam, and P. Shum, Appl. Phys. Lett. 90 (2007).

10. Z. Wu, Y. G. Liu, Z. Wang, M. Jiang, W. Ji, T. Han, S. Li, X. Shao, X. Q. Dinh, S. C. Tjin, and P. P. Shum, Opt. Lett. 38, 4070-4073 (2013).

11. J. Chen, Q. Liu, X. Fan, and Z. He, Opt. Lett. 41, 1066-1069 (2016).

12. H. Zhang, Z. Wu, P. P. Shum, X. Q. Dinh, C. W. Low, Z. Xu, R. Wang, X. Shao, S. Fu, and W. Tong, Scientific reports 7, 46633 (2017).

13. M. GRABK, B. Wajnchold, S. Pustelny, W. Gawlik, K. Skorupski, and P. Mergo, Acta Phys. Pol. A 118, 1127-1132 (2010).

14. E. Li, Opt. Lett. 32, 2064-2066 (2007).

15. G. Liu, M. Han, and W. Hou, Opt. Express 23, 7237-7247 (2015). 


\section{Full Reference}

1. K. Li, N. Zhang, N. M. Y. Zhang, et al. "Ultrasensitive measurement of gas refractive index using an optical nanofiber coupler," Optics letters, 2018, 43(4): 679-682.

2. N. Zhang, G. Humbert, T. Gong, et al. "Side-channel photonic crystal fiber for surface enhanced Raman scattering sensing," Sensors and Actuators B: Chemical, 2016, 223: 195-201.

3. Y. Zheng, Z. Wu, P. P. Shum, et al. "Sensing and lasing applications of whispering gallery mode microresonators," Opto-Electronic Review, 2018, 1(9): 18001501-18001510.

4. Y. Zheng, P. P. Shum, S. Liu, et al. "Experimental and numerical investigation on hollow core photonic crystal fiber based bending sensor," Optics express, 2019, 27(21): 30629-30638.

5. G. Liu, Q. Sheng, W. Hou, et al. "High-resolution, large dynamic range fiberoptic thermometer with cascaded Fabry-Perot cavities," Optics letters, 2016, 41(21): 5134-5137.

6. Q. Liu, T. Tokunaga, Z. He. "Ultra-high-resolution large-dynamic-range optical fiber static strain sensor using Pound-Drever-Hall technique," Optics letters, 2011, 36(20): 4044-4046.

7. X. W. Ye, Y. H. Su, J. P. Han. "Structural health monitoring of civil infrastructure using optical fiber sensing technology: A comprehensive review," The Scientific World Journal, 2014, 2014: 1-11.

8. T. Chan, L. Yu, H. Tam, et al. "Fiber Bragg grating sensors for structural health monitoring of Tsing Ma bridge: Background and experimental observation," Engineering structures, 2006, 28(5): 648-659.

9. X. Dong, H. Tam, P. Shum. "Temperature-insensitive strain sensor with polarization-maintaining photonic crystal fiber based Sagnac interferometer," Applied physics letters, 2007, 90(15): 151113.

10. Z. Wu, Y. Liu, Z. Wang, et al. "Simultaneous measurement of curvature and strain based on fiber Bragg grating in two-dimensional waveguide array fiber," Optics letters, 2013, 38(20): 4070-4073.

11. J. Chen, Q. Liu, X. Fan, et al. "Ultrahigh resolution optical fiber strain sensor using dual Pound-Drever-Hall feedback loops," Optics letters, 2016, 41(5): 1066-1069.

12. H. Zhang, Z. Wu, P. P. Shum et al. "Highly sensitive strain sensor based on helical structure combined with Mach-Zehnder interferometer in multicore fiber," Scientific reports, 2017, 7: 46633.

13. M. GRABK, B. Wajnchold, S. Pustelny, et al. "Experimental and Theoretical Study of Light Propagation in Suspended-Core Optical Fiber," Acta Physica Polonica A, 2010, 118(6): 1127-1132.

14. E. Li. "Temperature compensation of multimode-interference-based fiber devices," Optics letters, 2007, 32(14): 2064-2066.

15. G. Liu, M. Han, W. Hou. "High-resolution and fast-response fiber-optic temperature sensor using silicon Fabry-Pérot cavity," Optics express, 2015, 23(6): 7237-7247. 